\title{
Ambiguous Body OWNERShIP EXPERIENCE CAUSED BY FEELING-JUDGEMENT CONFLICT: EVIDENCE FROM SubJECTIVE MEASUREMENT IN RUBBER HAND ILLUSiON USING INTEGRATED INFORMATION THEORY
}

\section{A PREPRINT}

\author{
Takayuki Niizato* \\ Faculty of Engineering \\ Information and Systems \\ University of Tsukuba, Tsukuba \\ t_niizato@yahoo.co.jp \\ Kotaro Sakamoto \\ The Institute of Statistical Mathematics \\ Research Center for Statistical Machine Learning \\ xkotaro@ism.ac.jp
}

Tatsuya Okabayashi

Department of Information

and Management Systems Engineering

Nagaoka University of Technology, Niigata

\author{
Yuta Nishiyama* \\ Department of Information \\ and Management Systems Engineering \\ Nagaoka University of Technology, Niigata \\ y-nishiyama@kjs.nagaokaut.ac.jp \\ Takumi Kazama \\ Department of Information \\ and Management Systems Engineering \\ Nagaoka University of Technology, Niigata \\ Taiki Yamaguchi \\ Department of Information \\ and Management Systems Engineering \\ Nagaoka University of Technology, Niigata
}

November 30, 2021

\begin{abstract}
Human body awareness is malleable and adaptive to changing contexts. The illusory sense of bodyownership has been studied since the publication of the rubber hand illusion, where ambiguous body ownership feeling, expressed as "the dummy hand is my hand even though that is not true", was first defined. Phenomenologically, the ambiguous body ownership is attributed to a conflict between feeling and judgement; in other words, it characterises a discrepancy between first-person (i.e. bottom-up) and third-person (i.e. top-down) processes. Although Bayesian inference can explain this malleability of body image sufficiently, the theory does not provide a good illustration of why we have different experiences to the same stimuli - the difficulty lies in the uncertainty regarding the concept of judgement in their theory.

This study attempts to explain subjective experience during rubber hand illusions using integrated information theory (IIT). The integrated information $\Phi$ in IIT measures the difference between the entire system and its subsystems. This concept agrees with the phenomenological interpretation - that is, there is conflict between judgement and feeling. By analysing the seven nodes of a small bodybrain system, we demonstrate that the integrity of the entire system during the illusion decreases with increasing integrity of its subsystems. These general tendencies agree well with many brain-image analyses and subjective reports; furthermore, we found that subjective ratings were associated with the $\Phi$ s. Our result suggests that IIT can explain the general tendency of the sense of ownership illusions and individual differences in subjective experience during the illusions.
\end{abstract}

Keywords Sense of ownership · Rubber hand illusion · Integrated information theory · Subjective experience 


\section{Introduction}

The malleability of human body image shows that the perceptual system is not fixed but flexible. This malleability has been demonstrated in several experiments where the body awareness is altered depending on multimodal stimuli. One famous example is the rubber hand illusion (RHI) introduced in Botvinick and Cohen [1998], where subjects observe a brush stroking on a dummy hand while the subjects' real hand, which is hidden behind a partition, is also stroked. Synchronized visuo-tactile stimuli lead to an illusory sense of ownership over the dummy hand. The body-ownership over the dummy body-parts can be extended to the entire body. In Lenggenhager et al. [2007], for instance, the authors showed that a person observing the virtual body being touched while simultaneously the person is also touched caused an out-of-body experience. Interestingly, they found that the stimulation of specific brain regions, precisely the temporo-parietal junction area, could evoke a similar experience [Blanke and Arzy, 2005]. This region is known to work in consonance with multiple body sensations [Blanke et al. 2004]. Despite numerous studies conducted in this regard, a comprehensive understanding of the sense of ownership is still lacking.

Recent phenomenological interpretations provide a concise scheme for understanding the origin of bodyownership[Gallagher and Zahavi, 2007, De Vignemont, 2011. David and Ataria, 2021]. Ataria [2018] summarised the type of feeling-judgement conflict to clarify the various states of body-ownership. Phenomenologically, feelings involve closed sensor-motor loops; by contrast, judgements are more abstract reasoning processes. In summary, feelings give a first-person perspective, i.e. a non-conceptual, pre-reflective, and bottom-up process. By contrast, judgement gives a third-person perspective, i.e. observational, reflective, and top-down process. According to his argument, during an RHI, subjects feel the dummy hand as their hand but never admit (or judge) it as their hand. This conflict between feeling (i.e. this is my hand) and judgement (i.e. this is not my hand) causes ambiguous body ownership (ABO) rather than a specific sense of body-ownership [De Vignemont, 2011]. The fact that the results of the two processes are not necessarily consistent motivated us to consider that malleability of body image originates during the mismatched feeling-judgement process.

The free-energy principle (FEP) (or Bayesian inference) is a promising theory for understanding the flexibility of our perceptual system [Friston et al. 2014]. According to the FEP, we can change our beliefs whenever confronted with unexpected situations (i.e. high saliency). The perceptual system updates prior hypotheses with the more preferable posterior hypotheses. The FEP suggests that appropriately adjusting the hypotheses in response to the environment corresponds to the malleability of body image. The RHI is, therefore, related to the corruption of sensorimotor predictive cycles. Phenomenologically, this sensor-motor prediction error indicates a corruption of the feeling system. Most studies do not proceed further than this interpretation of corruption at the feeling level; however, as discussed earlier, the corruption of the feeling system does not provide an adequate explanation for $\mathrm{ABO}$ because we must also consider the top-down judgement level.

Tsakiris et al. [2011] investigated the interaction between bottom-up and top-down processes through interoceptive and exteroceptive perceptions [Apps and Tsakiris, 2014, de Vignemont and Alsmith, 2017]. They showed that the intensity of RHI is due to the mismatch between interoceptive and exteroceptive predictions. In their experiments, subjects who predicted their heartbeats more precisely experienced less illusion of ownership during the RHI compared with people who predicted their heartbeats less precisely [Tsakiris et al. 2011]. Based on their results, they speculated the existence of an interdependent relationship between interoceptive and exteroceptive predictions, i.e. high interoceptive saliency suppresses exteroceptive saliency, resulting in ABO (see also Tsakiris [2018]).

A remarkable relationship between RHI subjectivity and FEP was proposed by Tsakiris [2018]; however, FEP per se is fraught with an issue that is open to the following questions. (I) In FEP, judgement is a process for selecting the highest saliency among possible saliencies. As discussed in Ransom et al. [2020], these saliency selections cannot explain human affective behaviour for low saliency. This limitation implies that the saliency-driven explanation is insufficient for human experience. If we accept (I), then we agree that there is no conflict between feeling and judgement because the judgement system mechanically picks up the highest saliency; in this case, the intensity of RHI is only ascribed to the feeling system. If we do not accept (I), then we agree that the sensor-motor cycle holds both feeling and judgement; in this case, we neglect the essential difference between feeling and judgement owing to the compression of two different systems into one sensor-motor system.

Integrated information theory (IIT) is expected to explain human consciousness [Balduzzi and Tononi, 2009, Tononi] et al. 2016, Oizumi et al. 2016a b]. The basic concept of IIT is summarized in the following propositions: the degree of consciousness can be measured as the difference between the entire system and the sum of its parts; that is, the irreducibility of the entire system to its parts is the key to understanding the experience of consciousness [Balduzzi and Tononi, 2009, Tononi et al., 2016]. Notably, IIT also includes feeling and judgement in the perceptual process. Feeling corresponds to the local process and represents the information process of the subsystem (i.e. the subsystems of the system); judgement, in contrast, corresponds to the global process and represents the information process of the entire 
system. Importantly, IIT rejects any external evaluations as an explanation for consciousness; therefore, there is no superiority between feeling and judgement. In this sense, judgement maintains a parallel relationship with feeling in IIT in contrast to FEP. The difference between feeling and judgement is revealed only by subjective experience; thus, IIT can mathematically evaluate body malleability induced by the conflict between feeling and judgement.

In this study, we examined the relationship between subjective experience and objective bio-signals during an RHI using IIT. Although IIT is usually applied to the complex neural network in the brain, in this study, we picked up seven physiological data from the body (respiration, heartbeat, and skin conductance from body processes) and brain (EEG with electrodes on midline frontal $(\mathrm{Fz})$, vertex $(\mathrm{Cz})$, midline parietal $(\mathrm{Pz})$, and midline occipital $(\mathrm{Oz})$ regions for the brain process). We focused on these data for the following reasons. As highlighted by Tsakiris [2018], the interaction between interoceptive (body) and exteroceptive processes (brain) might explain the degree of ABO during RHI. It is not always necessary to examine the entire brain to estimate a subjective experience. Their result suggests that the degree of conflict between body and brain, rather than that of the entire brain process, is enough to estimate the subject's experiences during a stimulus .

\section{The basic concept of IIT}

Let us first explain IIT concepts for the sake of readers unfamiliar with IIT. Although many concepts exist in IIT, we only focus on three: minimum information partition (MIP in short), main complexes, and integrated information $\Phi$.

IIT deals with the intrinsic, rather than extrinsic, information; it only depends on inner variables. Typical information theories used in biology focus on the relation between external inputs and their results. In this setting, the system itself becomes a black box. The aim of IIT is to consider this black box in terms of "what the system is" rather than "what the system does" [Albantakis and Tononi, 2015]. The basic concept of IIT expresses mutual information between the past and current system's states; mathematically, this is expressed as $I(X(t-\tau) ; X(t))$.

However, this simple mutual information contains redundant information on the system's integrity, which should be measured as the system's irreducibility to its parts (subsystems, in IIT). The information obtained from the subsystems must be subtracted from the entire information $I(X(t-\tau) ; X(t))$. The rest of the information represents the system's integrity; therefore, the problem for IIT lies in the determination of the system partitions.

This study applies IIT as the mismatch decoding proposed by Oizumi et al. [2016a] because $\Phi^{*}$ satisfies the following properties: (i) Non-negativity: the lower bound of $\Phi^{*}$ is zero; (ii) sensitivity to noise correlation: $\Phi^{*}$ deals with external correlated noise [Mediano et al. 2019]; and (iii) applicability to continuous variables. Sometimes, the IIT as the mismatch decoding is classified as "IIT 2.0" to distinguish with IIT 3.0 [Oizumi et al., 2014, Tononi and Koch, 2015. Albantakis et al. 2019].

\subsection{Integrated information $\left(\Phi^{*}\right)$ and minimum information partition (MIP)}

As mentioned in the previous section, the mode of partitioning is essential to determining the system's integrity. The MIP is the system partition where the system's integrity is the minimum. The system's integrity in $\Phi^{*}$ is given by

$$
\Phi^{*}=I(X(t-\tau) ; X(t))-I^{*}\left[X ; \tau, \mathcal{P}_{S}\right]
$$

where $I(X(t-\tau) ; X(t))$ is the mutual information between the current $X(t)$ and past states $X(t-\tau) ; S$ is the set of all nodes of a given system; $\mathcal{P}_{S}$ is the set of all bi-partitions (total $2^{|S|}-1$ partitions); $I^{*}\left(X ; \tau, \mathcal{P}_{S}\right)$ is a "hypothetical" mutual information, indicating the mismatched decoding in the partitioned probability distribution. Precisely, $I^{*}\left(X ; \tau, \mathcal{P}_{S}\right)$ is given as the partition $\max _{\beta} \tilde{I}\left(\beta ; X, \tau, \mathcal{P}_{S}\right)$ that minimizes $\Phi^{*}$ (see Oizumi et al. 2016a, Mediano et al. 2019] for further details about this expression).

Because $\Phi^{*}$ depends on the partition $\pi\left(\in \mathcal{P}_{S}\right)$, the MIP is the partition that minimizes the integrated information.

$$
\pi_{\mathrm{MIP}}:=\arg \min _{\pi \in \mathcal{P}_{S}} \Phi^{*}(\pi)
$$

The integrated information of $\pi_{\mathrm{MIP}}$ is expressed as $\Phi^{*}\left(\pi_{\mathrm{MIP}}\right)$. We simply denote $\Phi^{*}\left(\pi_{\mathrm{MIP}}\right)$ as $\Phi_{\mathrm{MIP}}$. Notably, if $\Phi_{\text {MIP }}$ equals zero, the parts of the system are mutually independent; that is, there is no interaction between the parts. In this sense, $\Phi_{\mathrm{MIP}}$ characterises the system's irreducibility to its parts. 


\subsection{Main complexes}

Generally, we can compute $\Phi_{\text {MIP }}$ for any subsystem in the system, not only for the set $S$. We denote each $\Phi_{\text {MIP }}$ for a subsystem $T$ as $\Phi_{\mathrm{MIP}}^{T}$, where $T \subset S$. A 'complex' is a subsystem $C(\subset S)$, where $\Phi_{\mathrm{MIP}}^{C}>\Phi_{\mathrm{MIP}}^{T}$ for all supersets of $S$. From this definition, we can define the main complexes as those with the local maximum $\Phi_{\mathrm{MIP}}^{M}$.

Definition (Main complex): A main complex is a complex $M$ satisfying $\Phi_{\mathrm{MIP}}^{M}>\Phi_{\mathrm{MIP}}^{R}$ for any subsystems $R \subset M$.

The definition says that if two main complexes exist (say, $A$ and $B$ ), then they are exclusive. IIT researchers consider these main complexes to be the system's information core that might be related to our conscious experience. The validity of this assumption remains to be demonstrated, although there is no doubt that such information cores play a vital role in living systems [Marshall et al. 2017, Sheneman et al., 2019, Niizato et al. 2020].

\section{Application to RHI}

\subsection{Participants}

Twenty-two healthy male adults (mean age, 21.1 years; SD, 0.5) were recruited among the students at Nagaoka University of Technology. All participants gave written consent to participate after receiving an explanation of the procedures involved. The study was conducted according to the principles of the Declaration of Helsinki and was approved by the Ethics Committee of Nagaoka University of Technology.

\subsection{Procedure}

Participants sat on a chair with their left arm on a table and their palms facing down. The experimenter stood opposite the participant across the table. A realistic-looking left rubber hand was placed to the right of the participants' real left hand. The distance between the index finger of the rubber hand and the index finger of the real hand was $15 \mathrm{~cm}$. Two grey towels covered the space from the participants' shoulder to the participants' wrist and the proximal end of the artificial hand. A $45 \mathrm{~cm} \times 60 \mathrm{~cm}$ standing screen between the rubber and real hand prevented participants from viewing their own left hand (S1 Fig). Two experimental conditions were performed in our study: synchronous and asynchronous stroking condition. The experiments comprised two blocks corresponding to these conditions. The sequence of the blocks was randomized and counterbalanced across participants. Each block lasted for 15 min with a few minutes of inter-block intervals: 5-min rest periods (Rest Phase: Pre and Post) before and after a 5-min stroking period (Stimulus Phase). The rubber hand and unseen hand were stroked by two brushes either synchronously or asynchronously during the stroking period. The trained experimenter indicated the predetermined pattern and frequency (40 bpm) of stimulation using a metronome through an earphone. In the asynchronous condition, the dummy hand was touched immediately after touching the hidden real hand by the metronome; that is, every visual stimulus followed a tactile stimulus in the asynchronous condition. Therefore, the tactile stimulation was identical for both conditions. After the second rest period, the participants answered a questionnaire about their subjective experiences during the stroking period.

\subsection{Measurement}

Physiological Measurement Throughout each experimental block, electroencephalogram (EEG), electrocardiogram (ECG), respiration air flow (RESP), and electrodermal activity (EDA) were recorded using a bio-amplifier (Polymate Pro MP 6000, Miyuki Giken Corp., Tokyo, Japan) at a sampling rate of $250 \mathrm{~Hz}$ and with a 24-bit resolution, using a $50-\mathrm{Hz}$ notch filter. Especially, for the EEG, four signals $(\mathrm{Fz}, \mathrm{Cz}, \mathrm{Pz}, \mathrm{Oz})$ were measured at the locations of the four electrodes on the scalp according to the international 10-20 systems for measuring the four EEG signals $(\mathrm{Fz}, \mathrm{Cz}, \mathrm{Pz}$, $\mathrm{Oz}$ ); here, we used a high-frequency filter of $50 \mathrm{~Hz}$ and a low-frequency filter of $1 \mathrm{~Hz}$.

Questionnaire The questionnaire included nine statements from the original RHI study [Botvinick and Cohen, 1998] presented on a computer screen in a random order $\mathrm{S} 1 \mathrm{Table}$. Participants indicated their response by clicking on a visual-analogue scale ranging from strong agreement (100) to strong disagreement (0) including neither agreement nor disagreement (50). All the questions and instructions were presented in Japanese. Questionnaire data from one participant were excluded from the analysis because they were lost owing to mechanical issues.

\subsection{Data setting for IIT 2.0 application}

Kanai and Oizumi et al. proposed approximation methods for the computational problem of IIT [Oizumi et al. 2016b a, Kitazono et al. 2018, Hidaka and Oizumi, 2018]. This study applied their "Practical $\Phi$ Toolbox for MATLAB" to the 
physiological data; we considered only seven physiological data for the 22 subjects. The exhaustive method (computing all possible MIPs) can be applied in realistic computation time for such a small system.

Another parameter is time delay $\tau$ (IIT has only two constraints: partitions $\pi$ and time delay $\tau$ ). To choose the suitable parameter, we computed $\Phi_{\text {MIP }}$ for $\tau$ from 0 to 250 frames. The time delay $\tau$ is the point where the mean is maximum $\Phi_{\text {MIP }}$ for all subjects. We found that this $\tau$ is approximately 50 frames (i.e. $0.2 \mathrm{~s}$ ). This value seems to be suitable because the value is the same as the human response time $\mathrm{S} 2 \mathrm{Fig}$.

The time series of each raw data was 225000 frames (250 frames per second). Because we applied a 50 frame-delay $(0.2 \mathrm{~s})$ for the computation of IIT 2.0, sufficient data series for IIT 2.0 are required. We attempted 500 frames ( $2 \mathrm{~s}), 750$ frames ( $3 \mathrm{~s}$ ) and 1250 frames ( $5 \mathrm{~s}$ ) for IIT 2.0 computations - the time window shifts 250 frames each. The first blank data for some frames $(500,750,1250$ frames) were set at zero (only the first five steps at most for 1250 frames), which sum up to 900 data sets for each subject. The results for all frame samples were almost the same (S2 Table.

We extracted all measures related to integrated information for 900 steps ( $1 \mathrm{~s}$ for one step). The first third (from $0-300$ steps) and the last third (from 600 - 900 steps) correspond to the pre- and post-stimulus resting states. The middle section (from $300-600$ steps) is where the synchronous or asynchronous stimulus was applied. For all statistical tests, we detrended the obtained $\Phi$ time series because our focus was on the variation due to the stimulus for our rubber hand experiments. A negative value of $\Phi_{\mathrm{MIP}}$ is due to this treatment.

\section{Results}

\subsection{MIP (minimum information partition)}

We examined the average tendency of MIP during the experiments. As earlier discussed, the MIP gives two measures for estimating the system's state: (i) MIP cut, which divides the two subsystems by the weakest information flow; (ii) $\Phi_{\text {MIP }}$, which is the degree of information loss by the MIP cut. $\Phi_{\text {MIP }}$ is positive by definition, otherwise (i.e., if it were equal to 0 ), the two subsystems would be completely independent systems.

This study used seven data sets (i.e. respiration (Res), heartbeat (ECG), and skin conductance (EDA) for BODY; midline frontal $(\mathrm{Fz})$, vertex $(\mathrm{Cz})$, midline parietal $(\mathrm{Pz})$, and midline occipital $(\mathrm{Oz})$ region for BRAIN as EEG) for IIT analysis. We considered that these body-brain signals work as a system $(S=\{$ Res, ECG, EDA, Fz, Cz, Pz, Oz $\})$. If the rubber hand stimulus causes a difference in the system, the relation among these seven data sets will change. This difference also reflects the MIP information.

(A)

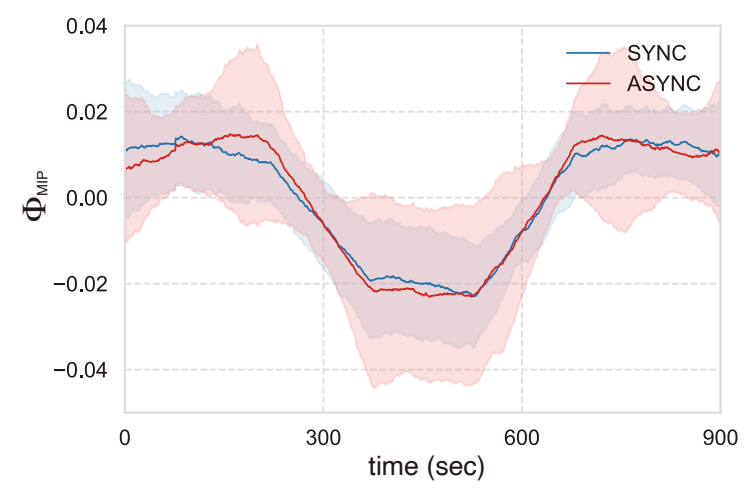

(B)

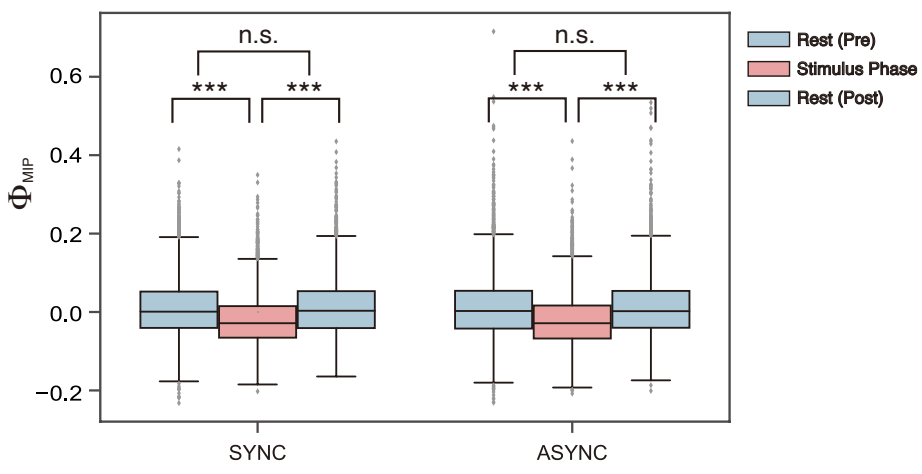

Figure 1: (A) Time series of mean $\Phi_{\text {MIP }}$ for both conditions (blue: SYNC, red: ASYNC). The stimulus phase is during $300 \backsim 600 \mathrm{~s}$. The other regions indicate resting states (no stimulus). The computational time frame for IIT is 500 (2 s). Moving-average (sample length 150 steps) was performed on the time-series for attaining easy to see temporal changes of values. (B) $\Phi_{\text {MIP }}$ of SYNC and ASYNC values drop significantly compared with each rest states $(* p<0.05$, $* * p<0.01, * * * p<0.005)$.

Figure 1 1 A shows the time series of the mean $\Phi_{\text {MIP }}$ for all 22 subjects. The $\Phi_{\text {MIP }}$ drops at the stimulus phase for both

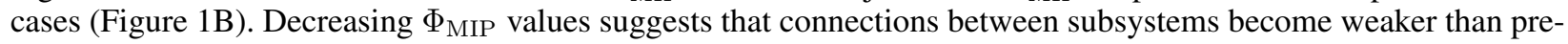
and post-stimulus. This result is interesting because similar changes of $\Phi_{\mathrm{MIP}}$ occur in both of SYNC and ASYNC conditions. 
(A)

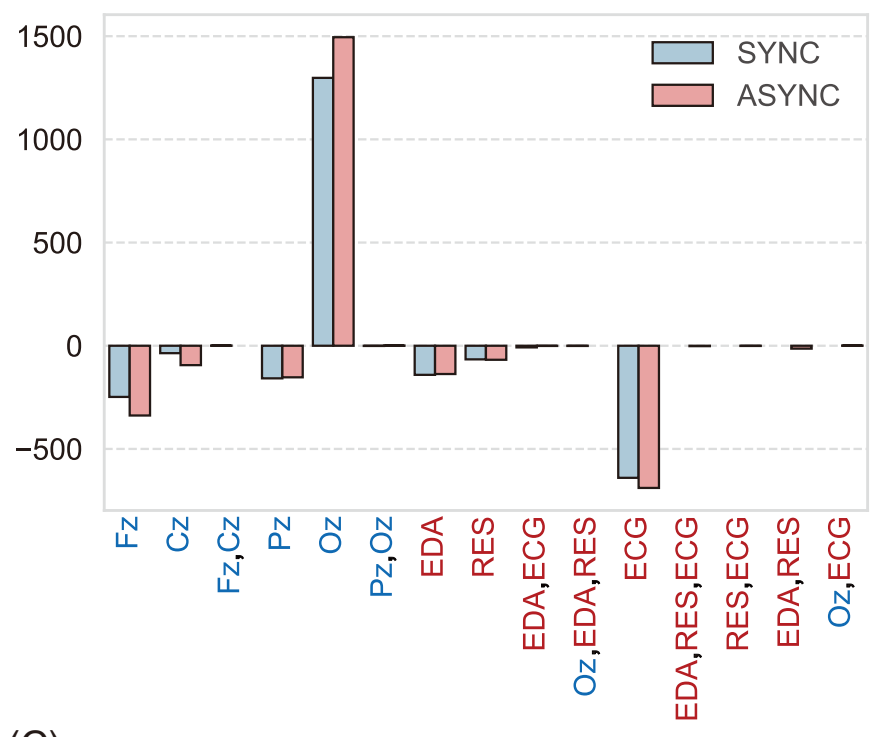

(B)

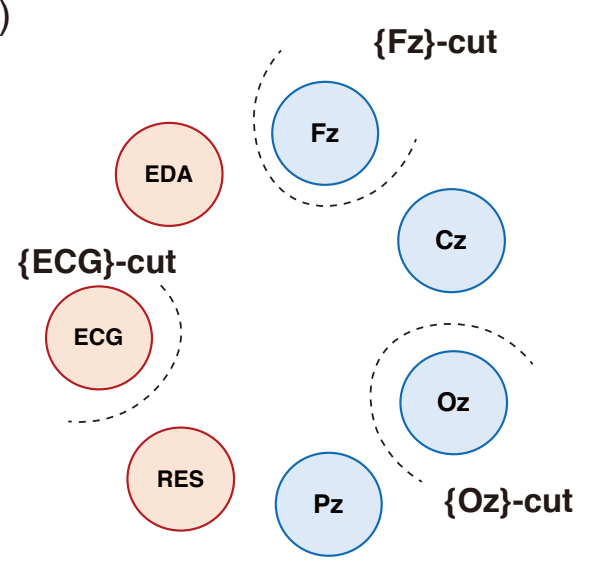

(C)

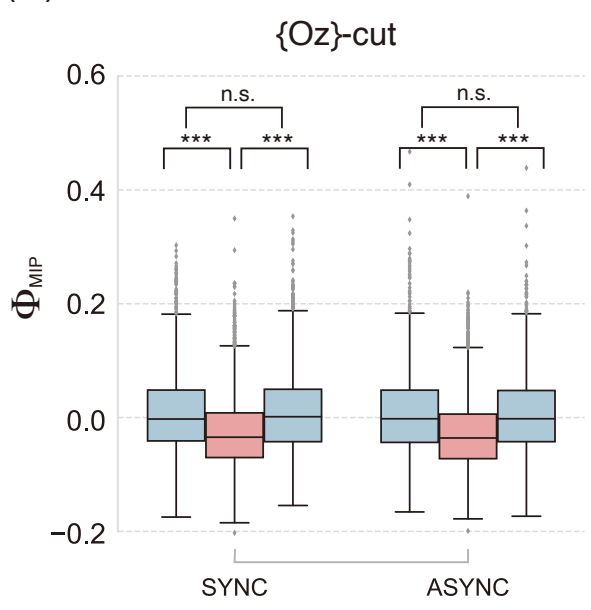

$\{\mathrm{Oz}\}$-cut

$\{F z\}$ cut
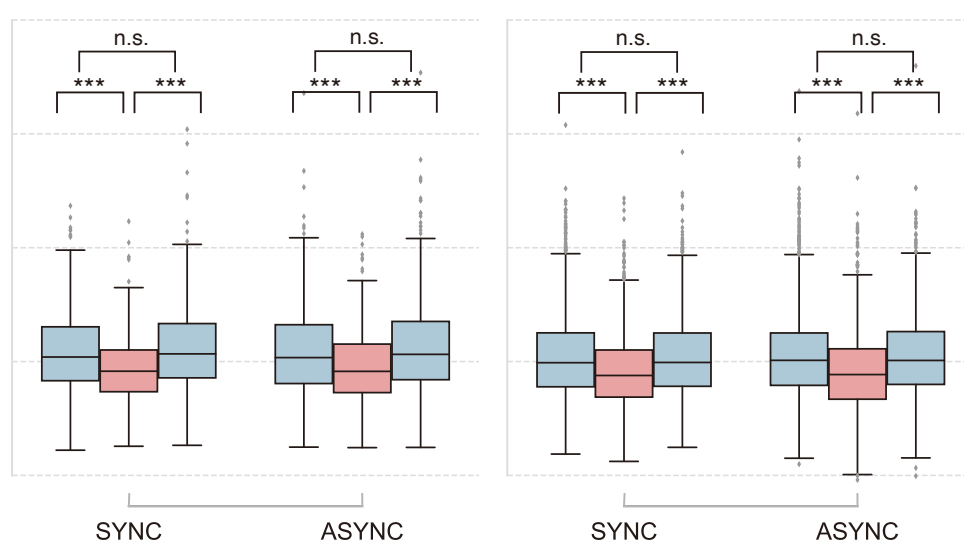

Figure 2: (A) Difference in the $\pi_{\mathrm{MIP}}$ frequency for pre- and intra -stimulus: $\operatorname{Fr}\left(\pi_{\mathrm{MIP}} \mid \mathrm{SP}\right)-\operatorname{Fr}\left(\pi_{\mathrm{MIP}} \mid \mathrm{PRE}\right)$. The computational time frame for IIT is $500(2 \mathrm{~s})$. The positive values indicate that a certain type of $\pi_{\text {MIP increases during }}$ the stimulus. Blue (red) bars show the frequency differences in the synchronous (asynchronous) condition. Blue (red) letters show data from the brain (body) system. (B) Image of MIP-cut location for the system $S .\{\mathrm{Oz}\}$-, $\{\mathrm{Fz}\}$-, $\{E C G\}$-cut are subsets with three largest differences shown in (A). (C) $\Phi_{\text {MIP }}$ for each MIP-cut location. For all cases, the $\Phi_{\mathrm{MIP}}$ drops during the stimulus $(* p<0.05, * * p<0.01, * * * p<0.005)$. No after-effects were observed.

Next, we examine the details of the MIP cut. The most remarkable tendency is the cut between $\{\mathrm{Oz}\}$ and $\{$ Res, ECG, $\mathrm{EDA}, \mathrm{Fz}, \mathrm{Cz}, \mathrm{Pz}$. More than $50 \%$ of MIP cuts were of this type (Figure 2A, B); furthermore, during the stimulus phase, the frequency of this cut increased statistically. Although their frequency increased, the $\Phi_{\text {MIP }}$ of $\{\mathrm{Oz}\}$-cut decreased. The implication of this tendency is suggestive. It is a well-known fact that the occipital region $(\mathrm{Oz})$ is involved in the visual information process. The high frequency of $\{\mathrm{Oz}\}$-cut reflects the discrepancy between visual information and tactile information. The low $\Phi_{\text {MIP }}$ during the stimulus means the visual process in Oz separates from other information processes (Figure $2 \mathrm{C}$ ).

For the remaining patterns, the MIP cut frequencies decreased after the stimulus was applied (Figure 2A, B). The decreased frequency of the rest compensates for the increased frequency of $\{\mathrm{Oz}\}$-cut. Interestingly, in the first case we observed, the cut was rarely located between the body and brain systems. For almost all cases, the cut located the brain system. This fact suggests that the body and the brain system is not the weakest link. The second most frequent $\Phi_{\text {MIP }}$ 
cut locates between $\{\mathrm{ECG}\}$ and $\{\mathrm{Res}, \mathrm{EDA}, \mathrm{Fz}, \mathrm{Cz}, \mathrm{Pz}, \mathrm{Oz}\}$. Taking account of this cut during stimulus, the heartbeat (ECG) merges into the cycle of the brain-body system during the stimulus. Even if $\{\mathrm{ECG}\}$ was selected as the cut, its integrity decreased during the stimulus. This aspect resonates with Tsakiri's result [Tsakiris et al., 2011].

To end this subsection, we highlight the following fact: $\Phi_{\text {MIP }}$ for SYNC and ANSYC condition during RHI shows the statistical difference between the two conditions observed (Welch's t test: $d f=13159$, $\mathrm{t}$-stat $=2.2561, p<0.05$ ). This result seems interesting because half of the subjects in our study reported a change of body ownership during both conditions. Many of the individual differences were not in nature but in degree. This logic also applies to the next subsection in Main complex analysis.

\subsection{Main complexes analysis}

We examine the average tendency of the main complexes for SYNC and ASYNC condition. The concept of the main complex accompanies some other concepts; namely, (i) $\Phi_{\mathrm{MIP}}^{T}$, which is the degree of information loss by partitioning the system into independent subsystems. The manner of partitioning system $T$ is determined by MIP: $\Phi_{\mathrm{MIP}}^{T}$; (ii) Complex, where all subsystems satisfy $\Phi_{\mathrm{MIP}}^{T}>0$ and $\Phi_{\mathrm{MIP}}^{T}>\Phi_{\mathrm{MIP}}^{R}$ for any superset $R$ of $T$; and (iii) Main complex, where the complex $M$ satisfies $\Phi_{\mathrm{MIP}}^{M}>\Phi_{\mathrm{MIP}}^{U}$ for any subset $U$ of $M$.

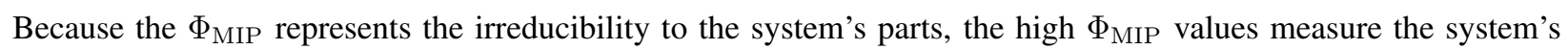
integrity. Notably, the system may include several main complexes. For a subsystem to be the main complex, it is sufficient that it does not contain other complexes (Lemma 4 in Kitazono et al. [2020]). In our analysis, the body-brain system has up to ten or more main complexes. The integrity of the system can be estimated as the sum of $\Phi_{\mathrm{MIP}} M$ of all main complexes $M$ in $S$ (denote $\sum_{M \in \mathcal{M}} \Phi_{\mathrm{MIP}}^{M}$ where $\mathcal{M}$ is a set of main complexes of system $S=\{$ Res, ECG, EDA, $\mathrm{Fz}, \mathrm{Cz}, \mathrm{Pz}, \mathrm{Oz}\})$.

(A)

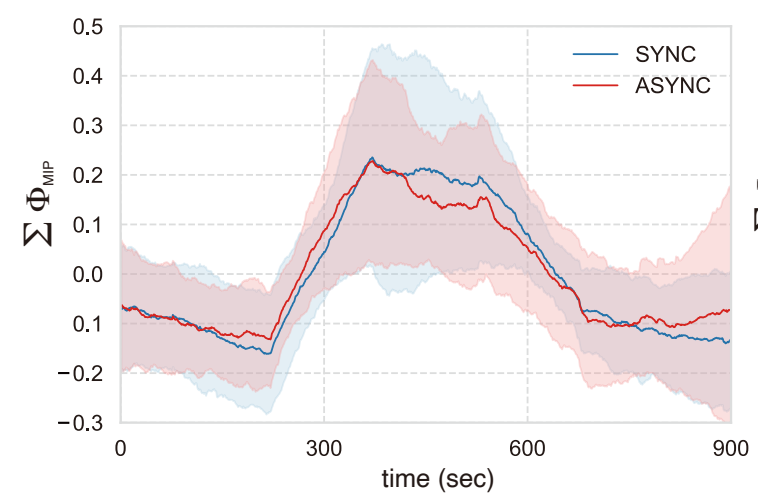

(B)

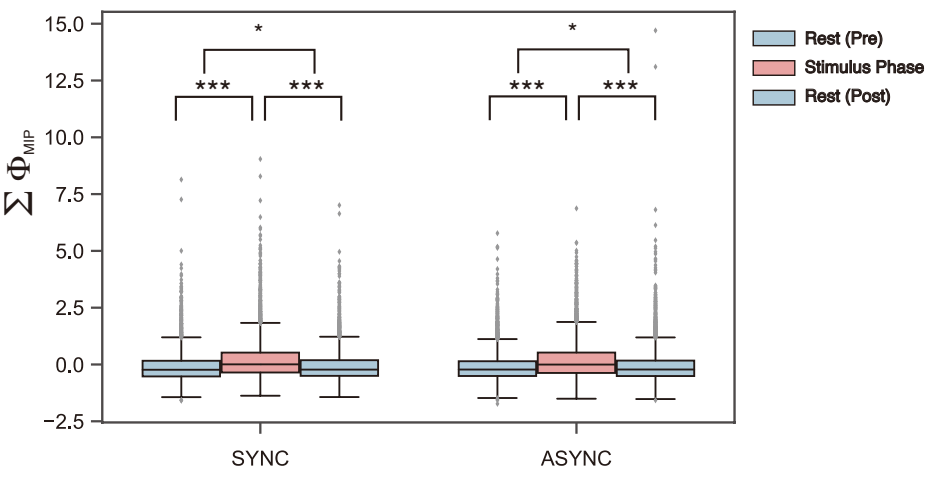

Figure 3: (A) Time series of mean $\sum_{M \in \mathcal{M}} \Phi_{\mathrm{MIP}}^{M}$ for both conditions (blue: SYNC, red: ASYNC). The stimulus phase is $300 \backsim 600 \mathrm{~s}$. The other regions are resting phase (no stimulus). The computational time frame for IIT is 500 ( $2 \mathrm{~s}$ ). Notably, the moving-average (sample length 150 steps) was performed on the time-series for attaining easy to see temporal changes of values. (B) $\sum_{M \in \mathcal{M}} \Phi_{\mathrm{MIP}}^{M}$ of SYNC and ASYNC values rises significantly compared with each rest phase $(* p<0.05, * * p<0.01, * * * p<0.005)$. In contrast to MIP analysis, an after-effect was observed for both conditions.

Figure $3 \mathrm{~A}$ shows that $\sum_{M \in \mathcal{M}} \Phi_{\mathrm{MIP}}^{M}$ increases only in the stimulus phase. In contrast to the decreasing $\Phi_{\mathrm{MIP}}^{S}$ for the entire system $S$, the integrities of the particular subsystems increased (Figure $3 \mathrm{~B}$ ). While the entire system splits up into relative independent parts, specific subsystems compensate by their integrity. This tendency can also be observed in the number of complexes. The number of irreducible subsystems (parts) increased during the stimulus phase (S4 Fig). Furthermore, in contrast to the $\Phi_{\mathrm{MIP}}^{S}$, an after-effect was observed in the main complex. This means that fluctuations in the system integrities remained even after the stimulus had ended.

The content of the most vital main complex (i.e. $\max _{M \in \mathcal{M}}\left\{\Phi_{\mathrm{MIP}}^{M}\right\}$ : the main complex $M$ with the highest $\Phi_{\mathrm{MIP}}^{M}$ ) is more suggestive. Figure 44 shows that the frequency of the main complex containing all elements (or $\Phi_{\text {MIP }}^{S}$ ) dropped during the stimulus phase. Here, the value of $\Phi_{\text {MIP }}^{S}$ indicates that the body-brain unit itself is irreducible to its parts. Metaphorically, the oneness of the body-brain system was corrupted during the stimulus. After the stimulus phase, the frequency of this main complex recovered. 
(A)

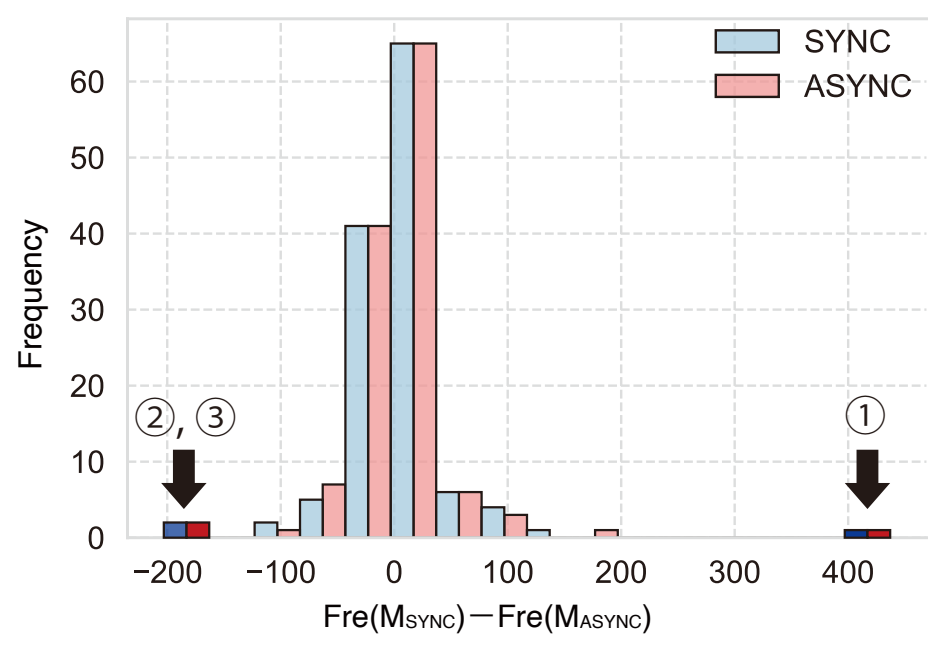

(B)
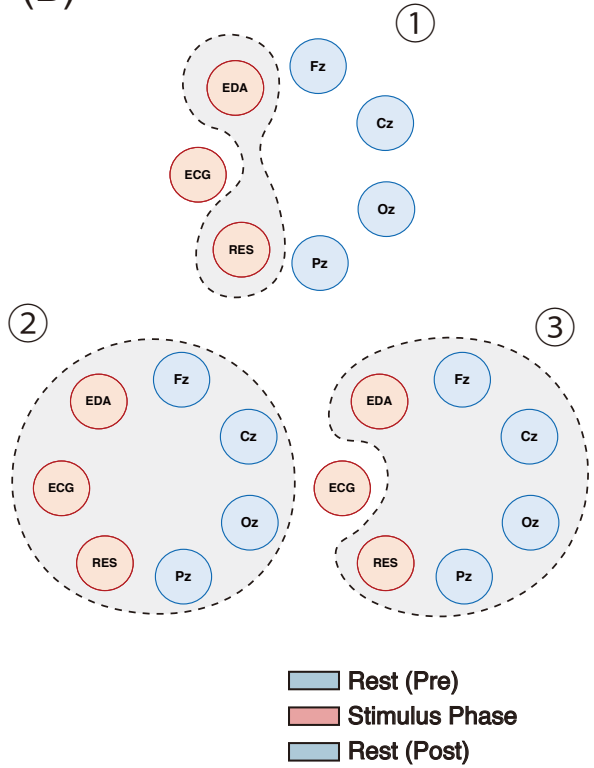

(C)

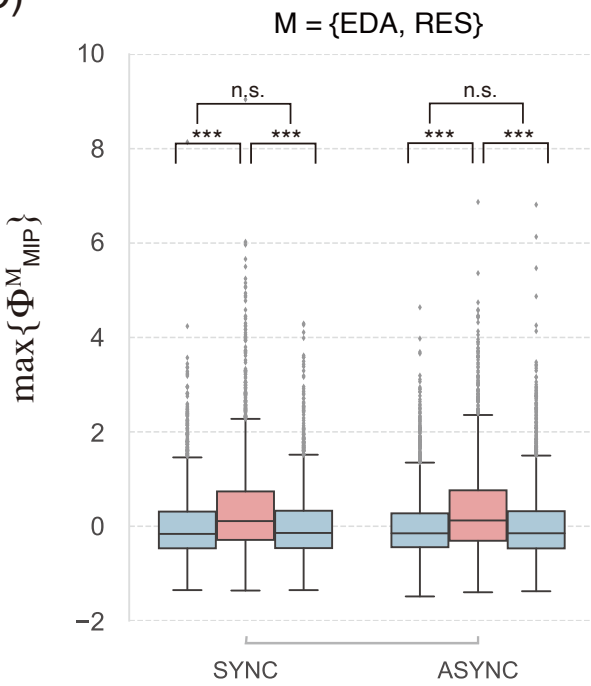

$M=S$

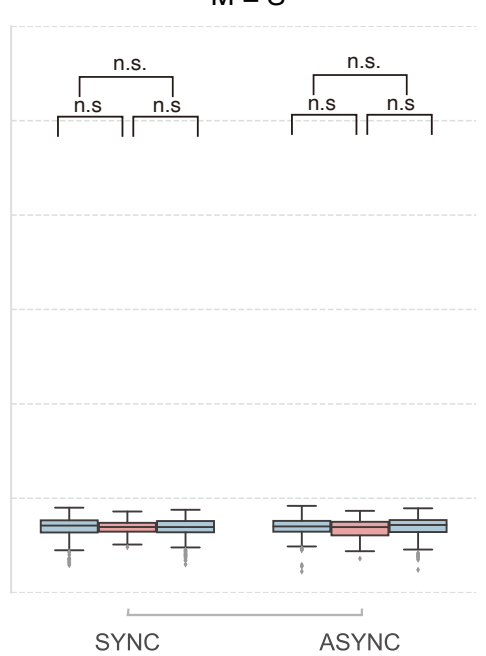

$M=S-\{E C G\}$
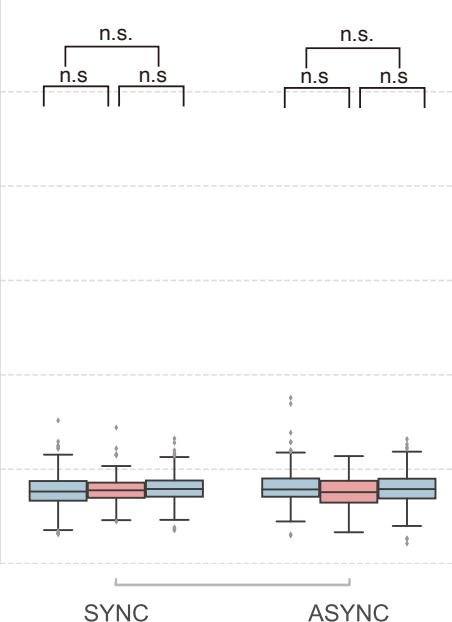

Figure 4: (A) Histogram of the difference of the $\max _{M \in \mathcal{M}}\left\{\Phi_{\text {MIP }}^{M}\right\}$ frequency for pre- and intra-stimulus: Fr $(M \in$ $\mathcal{M} \mid \mathrm{SP})-\operatorname{Fr}(M \in \mathcal{M} \mid \mathrm{PRE})$. Because many main complexes exist, we only analyse the outliers (indexed 1, 2, 3). Blue (red) bars show the frequency in the synchronous (asynchronous) condition. (B) Image of the main complex for the system $S$. $\{\mathrm{EDA}, \mathrm{RES}\}, S, S-\{\mathrm{ECG}\}$ are three highest differences. (C) $\max _{M \in \mathcal{M}}\left\{\Phi_{\mathrm{MIP}}^{M}\right\}$ for each main complex. Only $M=\{\mathrm{EDA}, \mathrm{RES}\}$, the $\Phi_{\mathrm{MIP}}$ rises significantly during the stimulus. The other two values are negative throughout three phases. $(* p<0.05, * * p<0.01, * * * p<0.005)$.

The other main complexes compensated for the decreasing frequencies of the complexes $S$ (Figure $4 \mathrm{~B}, \mathrm{C}$ ). The main complex $\{$ EDA, RES $\}$ is the most significant: skin conductance and respiration. The $\Phi_{\mathrm{MIP}}^{\{\mathrm{EDA}, \mathrm{RES}\}}$ also increased significantly during the stimulus (Figure 4C). The integrity only involves the body parts. Another high frequent main complex is $\{\mathrm{Fz}, \mathrm{Cz}, \mathrm{EDA}, \mathrm{RES}\}$, containing $\{\mathrm{Fz}, \mathrm{Cz}\}$ pairs. The frequency of this complex increased during the stimulus (the $\Phi_{\mathrm{MIP}}^{\{\mathrm{Fz}, \mathrm{Cz}, \mathrm{EDA}, \mathrm{RES}\}}$ also increased: Welch's t test: $d f=553$, t-stat $=-4.0359, p<10^{-6}$ ). This might relate to how the subject attempts to match past information and current information. 
As was observed in MIP analysis, $\sum_{M \in \mathcal{M}} \Phi_{\mathrm{MIP}}^{M}$ for the SYNC condition during the stimulus is statistically more significant than that of the ASYNC (Welch's t test: $d f=13177, \mathrm{t}-\mathrm{stat}=-2.3734, p<0.05$ ). Although both conditions changed the subject's body ownership, the $\Phi$ value reflected the difference between the two subjective reports of the participants.

\subsection{IIT 2.0 estimates the degree of ownership}

In this final subsection, we discuss how IIT 2.0 can estimate subjective reports of body ownership. The original purpose of IIT was to measure the degree of consciousness theoretically. Although the estimation of consciousness itself is still beyond our computational power, this aim could be attained through our small body-brain system.

We have thus far observed the average effects of (a-)synchronous stimulus on each brain-body system; however, as we have seen, there are individual differences in their effects (S3 Fig). While some subjects had a strong ownership illusion for their hand during the stimulus, some subjects did not. Some subjects had an ownership illusion for both conditions, while others only had it for the rubber hand condition. Although, statistically, SYNC subjects felt more intense ownership illusion compared with ASYNC subjects, it is desirable to know why such individual differences emerge.

We averaged the subjective values for Q1-Q3 questions, which indicate ABO for each subject, for both conditions to estimate the subjective reports. This simple computation suggests that the SYNC condition has a strong ownership illusion compared with the ASYNC condition. We use these mean values for each subject for the following analysis.

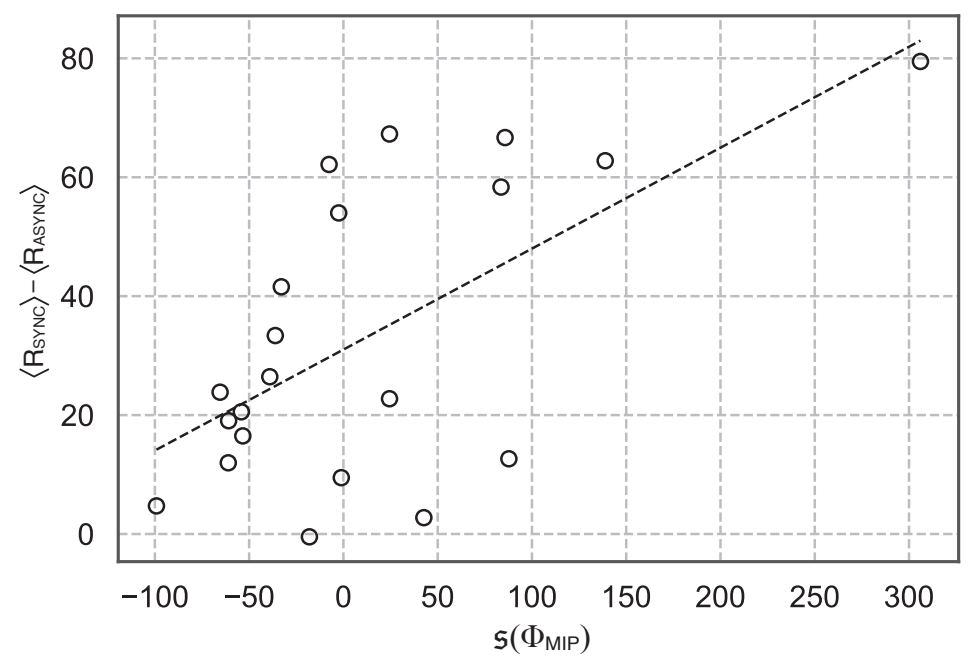

Figure 5: Relationship between the difference of mean subjective report $\left\langle R_{\mathrm{SYNC}}\right\rangle-\left\langle R_{\mathrm{ASYNC}}\right\rangle$ and $\mathfrak{s}\left(\Phi_{\mathrm{MIP}}\right)$ (see Eq. 3 ) for each subject. The correlation efficient is $r=0.56$ (Pearson's correlation test: $n=21, p<0.005$ )

We begin by answering the following question: can the integrated information and $\Phi^{*}$ for each subject account for such subjective individual differences? The answer is negative. For both conditions, $\sum \Phi_{\mathrm{MIP}}\left(\right.$ abbr. $\left.\sum_{M \in \mathcal{M}} \Phi_{\mathrm{MIP}}^{M}\right)$ shows low correlations (SYNC: Pearson's correlation test, $n=21, r=0.28, p=0.21$. ASYNC: $n=21, r=0.20$, $p=0.38$ ). Interestingly, $\sum \Phi_{\mathrm{MIP}}$ itself does not reflect the subjective reports. In our system, at least, the $\sum \Phi_{\mathrm{MIP}}$ is not the proper estimator of subjective reports.

We examine the difference in ownership intensities between the SYNC and the ASYNC condition. After subtracting the baseline (mean pre-stimulus phase for each subject), we take the difference of stimulus phase between the SYNC and the ASYNC. We define the difference in ABO intensities as the sum of this time series. Mathematically, this measure can be expressed for each subject indexed $i$ :

$$
\mathfrak{s}_{i}\left(\Phi_{\mathrm{MIP}}\right)=\sum_{t=300}^{600}\left\{\sum_{M \in \mathcal{M}_{t}}\left(\bar{\Phi}_{\mathrm{MIP}}^{M, \mathrm{SYNC}}-\bar{\Phi}_{\mathrm{MIP}}^{M, \mathrm{ASYNC}}\right)\right\}
$$

where $\bar{\Phi}_{\mathrm{MIP}}^{M, \mathrm{SYNC}}$ means that the integrity of main complex $M$ at time $t$ for the SYNC condition subtracted the baseline. 
Figure 5 shows the correlation between the subjective difference and ownership intensities (Pearson's correlation test: $n=21, r=0.56, p<0.005)$. The gap between the two experimental settings can comprehend the subjective difference for their ABO. Our result shows that the $\Phi_{\text {MIP }}$ gaps, rather than of $\Phi_{\text {MIP }}$ itself, can estimate the subjective individual differences for the different experimental settings.

\section{Discussion}

Throughout this study, we have examined two aspects of $\mathrm{ABO}$ induced by the rubber hand stimulus: the average tendency and the individual difference.

\subsection{Average tendencies for (a-)synchronous stimulus of the rubber hand}

Applying IIT 2.0 to the small body-brain system, we found some new insights. The minimum information partition (MIP) reveals that the system's independence emerges during the stimulus for both experimental conditions. The location of the MIP cut indicates that the disjunctive parts are not body-brain pairs but intra-brain or intra-body pairs. The main complex analysis gives this trend a more precise picture - the frequency of all-elements main complex $S$ decreases during the stimulus phase. Before the stimulus, the body-brain system works as one irreducible system. Although the main complex of all elements $S$ exists even in the stimulus phase, the degree of this irreducibility $\left(\Phi_{\text {MIP }}^{S}\right)$ decreases. These results agree with our intuition on these experimental settings.

The main complex shows high integrities (i.e. $\sum \Phi_{\mathrm{MIP}}$ ) during the stimulus on average. The disconnected system in terms of MIP compensates for its disconnection by increasing the integrity of its parts. The high irreducible parts, for example, contain Fz, Cz, Res, ECG. Considering that MIP locates at Oz, the complexes, including Fz and Cz, also have a compensated meaning. In the stimulus phase, the brain might raise its integrity for the working memory (Fz) and motor function $(\mathrm{Cz})$ instead of separating the inconsistent visual information $(\mathrm{Oz})$. This relation makes sense if we consider that the motor-perception with the subject's short term memory (including multisensory integration at the ventral premotor cortex [Ehrsson et al. 2004, Gentile et al., 2015]) attempts to counterbalance the mismatch in the visual system. The body integrity (Res and EDA) is also observed in the stimulus phase. Notably ECG (i.e. heartbeat) is excluded from this body-integrity (the MIP cut locates at $\{E C G\}$ in Figure $2 B$ ). This relative independency of the heartbeat might be related to Tsakiris et al. [2011] results.

Although there were some differences between SYNC and ASYNC conditions, we also observed considerable similari-

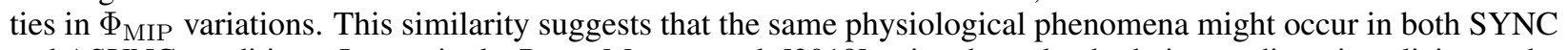
and ASYNC conditions. Interestingly, Perez-Marcos et al. [2018] pointed out that body image distortion elicits can be elicited by ASYNC condition, but not SYNC condition. Their results imply that the ASYNC condition also changes latent body cognition, and our analysis can associate with their results.

\subsection{Individual differences of $\mathrm{ABO}$ with IIT 2.0}

Although our average IIT analysis succeeded in explaining the general tendencies, individual differences still exist. Some subjects experience more ABO than ASYNC condition; others experience ABO as the same as the same condition. Previous studies try to explain these various individual differences from the physiological perspective [Armel and Ramachandran, 2003]; however, there are no consistent conclusions.

We found that the $\sum \Phi_{\text {MIP }}$ value itself does not reflect subjective reports. Instead, the difference between them correlates the subjective reports. This result suggests that subjective evaluation is not determined by a single experience but rather by comparing different experiences. We also attempted the same analysis applying the peak-end rule, which states that how they felt at its peak and at its end determines judgement on a given experience [Kahneman et al. [1993]. The result was the same.

Why does the difference in $\Phi_{\text {MIP }}$ correlate with individual subjective report? As discussed in our result, this might be due to the manner of reporting. The subject was required to answer their feelings of $\mathrm{ABO}$ from $0-100^{\circ}$. Almost all subjects would have been asked this question for the first time. This setting means that the subject cannot access his or her past ownership experience. The meaning of ownership attains its definitive status after comparing the different types of ownership. Without such different experiences, the subjective report has no reference point. Therefore, the anchoring effect of the subjective report might be cancelled out by considering differences between two different ownership experience . 


\subsection{Evaluate subjective experience: free energy and integrated information}

Sometimes, the rubber hand illusion has been interpreted in the context of Bayesian inference [Samad et al. 2015. Rood et al. 2020]. Bayesian inference says that the subject attempts to eliminate perceptual inconsistency by replacing it with a new hypothesis. This assumption agrees with the free energy principle (FEP) proposed by Friston. Because perceptual mismatch increases the system's free energy (or surprise), it attempts to decrease it with an updated hypothesis.

How does this FEP relate to IIT? A possible answer is that the relation between FEP and IIT is that they are "two sides of the same coin'. Some simulation studies help us with this understanding. Although there are many simulation studies on IIT, almost all of them make the same observation; that is, the integrated information mainly increases in the learning phase [Albantakis et al. 2014, Abrego and Zaikin, 2019, Sheneman et al., 2019]. Once the order of the system has been established, the $\Phi$ values decrease [Sheneman et al., 2019].

If we observe that the $\Phi$ originally refers to consciousness, these tendencies suggest much awareness needed during the learning phase. However, Solms and Friston [2018] have indicated that reducing "surprise" means losing the conscious process (establishing the automatic response to the external environment [Solms, 2019]). In this sense, we might say that the IIT and FEP observe the same object from different perspectives.

In this context, the RH stimulus might trigger a learning phase from the IIT perspective. From the point of view of the FEP, this also refers to the generation of a system's "surprise"; however, the process is not straightforward. As earlier noted, FEP hardly explains the conflict between feeling and judgement on sense of ownership by itself; IIT can access this conflict. Furthermore, our IIT analysis shows that the $\Phi$ value in IIT also fails to capture subjective experiences.

How can we relate our results to the subjective reports? Our small system cannot explain that consciousness itself might be one of the possible interpretations. Nevertheless, the most plausible interpretation lies in the process of subjective feeling estimation-, that is, the subject is required to report their feelings after each experiment. In this setting, the subject reports their judgement of experience rather than their ownership experience themselves. Judgement is not the direct experience, but an indirect experience mediated by language. The subjective report does not reflect subjective experiences directly (note that this judgement differs from the one we used in the feeling-judgement conflict; rather, it is the second-order judgement for this conflict).

There are many criticisms of IIT. One of the main criticisms stems from the statement that $\Phi$ can measure raw consciousness [Cerullo, 2015, Lombardi and López, 2018]. Indeed, this type of statement contains complex philosophical issues, which are not easy to solve. However, if we consider IIT as a measure of subjective experience on the second-order judgement level, then our study suggests that IIT may provide the mathematical framework for phenomenological descriptions of subjective experience.

\section{Supporting information}

S1 Fig. Experimental set-up in the stimulus phase. A dummy left hand was placed in front of the participants. The participants' left hand was hidden out of view. The right hand with a probe to measure EDA rested on a table. EEG electrodes were installed on participants' scalp. In addition, participants were equipped with ECG electrodes and nostril cannula to measure their respiration flow.

S2 Fig. $\Phi_{\text {MIP }}$ values along time delay $\tau$ s for the entire system $S$. The frame size is $900 \mathrm{~s}$. $\Phi_{\text {MIP }}$ peak locates at 0.2 s.

S3 Fig. Boxplot of rating scores for each questionnaire item. The data is available on S1 Table,

S4 Fig. Boxplot of the number of complexes for each main complex. The tendency is the same as the $\Phi_{\mathrm{MIP}}^{M}$ $(M \in \mathcal{M})$ distribution in Figure 4.

S1 Table. Questionnaire items [Botvinick and Cohen, 1998].

S2 Table. All statistical tests for each condition.

\section{References}

Matthew Botvinick and Jonathan Cohen. Rubber hands 'feel'touch that eyes see. Nature, 391(6669):756-756, 1998. doi:10.1038/35784. URL https://doi.org/10.1038/35784. 
bioRxiv preprint doi: https://doi.org/10.1101/2021.11.29.470324; this version posted December $1,2021$. The copyright holder for this preprint (which was not certified by peer review) is the author/funder, who has granted bioRxiv a license to display the preprint in perpetuity. It is made available under aCC-BY-NC-ND 4.0 International license.

Ambiguous Body Ownership Experience Caused by Feeling-judgement Conflict

A PREPRINT

Table 1: Questionnaire items [Botvinick and Cohen, 1998].

Q1 It seemed as if I felt the touch of a paintbrush in the location where I saw the dummy hand touched.

Q2 It seemed as if the touch I felt was caused by the paintbrush stroking the dummy hand.

Q3 I felt as if the dummy hand was my hand.

Q4 I felt as if my real hand was drifting towards the dummy hand.

Q5 It seemed as if I might have more than one left hand.

Q6 It seemed as if the touch I felt emanated from somewhere between my real hand and the dummy hand.

Q7 I felt as if my real hand was evolving into the dummy hand.

Q8 It appeared as if the dummy hand was drifting towards my real hand.

Q9 The dummy hand appeared to resemble my real hand visually.

Bigna Lenggenhager, Tej Tadi, Thomas Metzinger, and Olaf Blanke. Video ergo sum: manipulating bodily selfconsciousness. Science, 317(5841):1096-1099, 2007.

Olaf Blanke and Shahar Arzy. The out-of-body experience: disturbed self-processing at the temporo-parietal junction. The Neuroscientist, 11(1):16-24, 2005.

Olaf Blanke, Theodor Landis, Laurent Spinelli, and Margitta Seeck. Out-of-body experience and autoscopy of neurological origin, 2004. ISSN 00068950.

Shaun Gallagher and Dan Zahavi. The phenomenological mind: An introduction to philosophy of mind and cognitive science. 2007. ISBN 0203086597. doi:10.4324/9780203086599.

Frédérique De Vignemont. Embodiment, ownership and disownership. Consciousness and Cognition, 2011. ISSN 10902376. doi:10.1016/j.concog.2010.09.004.

Aviya Ben David and Yochai Ataria. The body image-body schema/ownership-agency model for pathologies: four case studies. In Body Schema and Body Image. 2021. doi:10.1093/oso/9780198851721.003.0020.

Yochai Ataria. Body disownership in complex posttraumatic stress disorder. 2018. ISBN 9781349953660. doi $10.1057 / 978-1-349-95366-0$

Karl J. Friston, Klaas Enno Stephan, Read Montague, and Raymond J. Dolan. Computational psychiatry: The brain as a phantastic organ, 2014. ISSN 22150374.

Manos Tsakiris, Ana Tajadura-Jiménez, and Marcello Costantini. Just a heartbeat away from one's body:Interoceptive sensitivity predicts malleability of body-representations. Proceedings of the Royal Society B: Biological Sciences, 2011. ISSN 09628452. doi:10.1098/rspb.2010.2547.

Matthew A.J. Apps and Manos Tsakiris. The free-energy self: A predictive coding account of self-recognition, 2014. ISSN 18737528.

Frédérique de Vignemont and Adrian Alsmith. The Subject's Matter. Self Consciousness and the Body. 2017. ISBN 9780262036832.

M. Tsakiris. The Material Me: Unifying the Exteroceptive and Interoceptive Sides of the Bodily Self. In The Subject's Matter. 2018. doi:10.7551/mitpress/10462.003.0019

Madeleine Ransom, Sina Fazelpour, Jelena Markovic, James Kryklywy, Evan T. Thompson, and Rebecca M. Todd. Affect-biased attention and predictive processing. Cognition, 2020. ISSN 18737838. doi $10.1016 /$ j.cognition.2020.104370

David Balduzzi and Giulio Tononi. Qualia: The geometry of integrated information. PLoS Computational Biology, 2009. ISSN 1553734X. doi 10.1371 /journal.pcbi.1000462

Giulio Tononi, Melanie Boly, Marcello Massimini, and Christof Koch. Integrated information theory: From consciousness to its physical substrate, 2016. ISSN 14710048.

Masafumi Oizumi, Shun-ichi Amari, Toru Yanagawa, Naotaka Fujii, and Naotsugu Tsuchiya. Measuring Integrated Information from the Decoding Perspective. PLOS Computational Biology, 12(1):1-18, $2016 \mathrm{.}$. doi:10.1371/journal.pcbi.1004654 URL/https://doi.org/10.1371/journal.pcbi.1004654.

Masafumi Oizumi, Naotsugu Tsuchiya, and Shun Ichi Amari. Unified framework for information integration based on information geometry. Proceedings of the National Academy of Sciences of the United States of America, 2016b. ISSN 10916490. doi:10.1073/pnas.1603583113.

Larissa Albantakis and Giulio Tononi. The intrinsic cause-effect power of discrete dynamical systems-from elementary cellular automata to adapting animats. Entropy, 2015. ISSN 10994300. doi:10.3390/e17085472. 
bioRxiv preprint doi: https://doi.org/10.1101/2021.11.29.470324; this version posted December $1,2021$. The copyright holder for this preprint (which was not certified by peer review) is the author/funder, who has granted bioRxiv a license to display the preprint in perpetuity. It is made available under aCC-BY-NC-ND 4.0 International license.

Ambiguous Body Ownership Experience Caused by Feeling-judgement Conflict

A PREPRINT

Pedro A.M. Mediano, Anil K. Seth, and Adam B. Barrett. Measuring integrated information: Comparison of candidate measures in theory and simulation. Entropy, 2019. ISSN 10994300. doi:10.3390/e21010017.

Masafumi Oizumi, Larissa Albantakis, and Giulio Tononi. From the Phenomenology to the Mechanisms of Consciousness: Integrated Information Theory 3.0. PLoS Computational Biology, 2014. ISSN 15537358. doi $10.1371 /$ journal.pcbi.1003588

Giulio Tononi and Christof Koch. Consciousness: Here, there and everywhere?, 2015. ISSN 14712970.

Larissa Albantakis, William Marshall, Erik Hoel, and Giulio Tononi. What caused what? A quantitative account of actual causation using dynamical causal networks. Entropy, 2019. ISSN 10994300. doi $10.3390 / \mathrm{e} 21050459$

William Marshall, Hyunju Kim, Sara I. Walker, Giulio Tononi, and Larissa Albantakis. How causal analysis can reveal autonomy in models of biological systems. Philosophical Transactions of the Royal Society A: Mathematical, Physical and Engineering Sciences, 2017. ISSN 1364503X. doi:10.1098/rsta.2016.0358

Leigh Sheneman, Jory Schossau, and Arend Hintze. The evolution of neuroplasticity and the effect on integrated information. Entropy, 2019. ISSN 10994300. doi $10.3390 / \mathrm{e} 21050524$.

Takayuki Niizato, Kotaro Sakamoto, Yoh ichi Mototake, Hisashi Murakami, Takenori Tomaru, Tomotaro Hoshika, and Toshiki Fukushima. Finding continuity and discontinuity in fish schools via integrated information theory. PLoS ONE, 2020. ISSN 19326203. doi:10.1371/journal.pone.0229573

Jun Kitazono, Ryota Kanai, and Masafumi Oizumi. Efficient algorithms for searching the minimum information partition in Integrated Information Theory. Entropy, 2018. ISSN 10994300. doi 10.3390/e20030173.

Shohei Hidaka and Masafumi Oizumi. Fast and exact search for the partition with minimal information loss. PLoS ONE, 2018. ISSN 19326203. doi:10.1371/journal.pone.0201126

Jun Kitazono, Ryota Kanai, and Masafumi Oizumi. Efficient search for informational cores in complex systems: Application to brain networks. Neural Networks, 2020. ISSN 18792782. doi 10.1016/j.neunet.2020.08.020.

H. Henrik Ehrsson, Charles Spence, and Richard E. Passingham. That's my hand! Activity in premotor cortex reflects feeling of ownership of a limb. Science, 2004. ISSN 00368075. doi:10.1126/science.1097011.

Giovanni Gentile, Malin Björnsdotter, Valeria I. Petkova, Zakaryah Abdulkarim, and H. Henrik Ehrsson. Patterns of neural activity in the human ventral premotor cortex reflect a whole-body multisensory percept. NeuroImage, 2015. ISSN 10959572. doi $10.1016 /$ j.neuroimage.2015.01.008.

Daniel Perez-Marcos, Matteo Martini, Christina T. Fuentes, Anna I. Bellido Rivas, Patrick Haggard, and Maria V. Sanchez-Vives. Selective distortion of body image by asynchronous visuotactile stimulation. Body Image, 2018. ISSN 17401445. doi $10.1016 /$ j.bodyim.2017.11.002.

K. Carrie Armel and V. S. Ramachandran. Projecting sensations to external objects: Evidence from skin conductance response. Proceedings of the Royal Society B: Biological Sciences, 2003. ISSN 14712970. doi 10.1098/rspb.2003.2364.

Daniel Kahneman, Barbara L. Fredrickson, Charles A. Schreiber, and Donald A. Redelmeier. When More Pain Is Preferred to Less: Adding a Better End. Psychological Science, 1993. ISSN 14679280. doi $10.1111 / \mathrm{j} .1467-$ 9280.1993.tb00589.x.

Majed Samad, Albert Jin Chung, and Ladan Shams. Perception of body ownership is driven by Bayesian sensory inference. PLoS ONE, 2015. ISSN 19326203. doi $10.1371 /$ journal.pone.0117178

Thomas Rood, Marcel van Gerven, and Pablo Lanillos. A deep active inference model of the rubber-hand illusion. In Communications in Computer and Information Science, 2020. ISBN 9783030649180. doi:10.1007/978-3-03064919-7_10.

Larissa Albantakis, Arend Hintze, Christof Koch, Christoph Adami, and Giulio Tononi. Evolution of Integrated Causal Structures in Animats Exposed to Environments of Increasing Complexity. PLoS Computational Biology, 2014. ISSN 15537358. doi:10.1371/journal.pcbi.1003966.

Luis Abrego and Alexey Zaikin. Integrated information as a measure of cognitive processes in coupled genetic repressilators. Entropy, 2019. ISSN 10994300. doi $10.3390 / \mathrm{e} 21040382$

Mark Solms and Karl Friston. How and why consciousness arises: Some considerations from physics and physiology. Journal of Consciousness Studies, 2018. ISSN 13558250.

Mark Solms. The hard problem of consciousness and the free energy principle. Frontiers in Psychology, 2019. ISSN 16641078. doi $10.3389 /$ fpsyg.2018.02714

Michael A. Cerullo. The Problem with Phi: A Critique of Integrated Information Theory. PLoS Computational Biology, 2015. ISSN 15537358. doi:10.1371/journal.pcbi.1004286.

Olimpia Lombardi and Cristian López. What does 'information' mean in integrated information theory? Entropy, 2018. ISSN 10994300. doi $10.3390 / \mathrm{e} 20120894$. 